Proceedings of the 46th International School and Conference on the Physics of Semiconductors "Jaszowiec" 2017, Szczyrk

\title{
InAs on InP Quantum Dashes as Single Photon Emitters at the Second Telecommunication Window: Optical, Kinetic, and Excitonic Properties
}

\author{
P. Mrowiński ${ }^{a}$, Ł. Dusanowski ${ }^{a, b}$, A. Somers ${ }^{b}$, S. Höfling ${ }^{b, c}$, J.P. Reithmaier ${ }^{d, e}$, \\ J. MisieWICZ ${ }^{a}$ AND G. SĘK ${ }^{a, *}$ \\ ${ }^{a}$ OSN Lab, Department of Experimental Physics, Faculty of Fundamental Problems of Technology, Wrocław \\ University of Science Technology, Wrocław, Poland \\ ${ }^{b}$ Technische Physik \& W.C. Röntgen-Center for Complex Material Systems, Universität Würzburg, Germany \\ ${ }^{c}$ School of Physics and Astronomy, University of St. Andrews, St. Andrews, United Kingdom \\ ${ }^{d}$ Institute of Nanostructure Technologies and Analytics, Technische Physik, Universität Kassel, Germany \\ ${ }^{e}$ Institute of Nanostructure Technologies and Analytics (INA), CINSaT, University of Kassel, \\ Heinrich-Plett-Str. 40, 34132 Kassel, Germany
}

\begin{abstract}
In this work, InAs/InGaAlAs/InP quantum dashes have been investigated in terms of their optical, kinetic, and excitonic properties with respect to their application within the $1300 \pm 40 \mathrm{~nm}$ spectral range, i.e. the Oband of the telecommunication technologies. We focused on the basic excitonic complexes such as neutral exciton, biexciton, and charged exciton, which have been identified by means of photoluminescence measurements. Emission and carriers' dynamics have been analyzed using rate equation model and fitting the experimental data obtained for both continuous-wave and pulsed excitation regimes. There has been found a significant impact of the charge carrier imbalance in the system and electron capturing rate on the dynamics of the optical and electronic transitions, which results in high occupation of the negatively charged trion state. Autocorrelation measurements show clear antibunching of trion emission for non-resonant excitation which indicates a potential of such kind of emitters as single photon sources for short-range quantum communication schemes.
\end{abstract}

DOI: 10.12693/APhysPolA.132.382

PACS/topics: photoluminescence, quantum dash, excitonic complexes, antibunching, autocorrelation, single photon source, telecommunication, time-resolved

\section{Introduction}

Security of the data transfer in contemporary communication is a key element that provides a stable economy and services. Quantum communication protocols that use single photon as an information carrier provide such security due to non-classical nature of quantum state that exclude the possibility of copying it unless being noticed. This motivated a great effort which has been focused on developing efficient quantum emitters based on semiconductor nanostructures that can deliver single photons on demand [1-3] or entangled photon pairs [4-6]. A step forward is to realize a single photon source coupled with an optical fiber to extend these schemes to the telecommunication networks, i.e. to match to the low loss spectral ranges at $1.3 \mu \mathrm{m}$ or $1.55 \mu \mathrm{m}$ wavelengths.

In this work we focus on the InAs/InGaAlAs/InP nanostructures which are of high interest due to a tunable emission range from 1.2 to $2.0 \mu \mathrm{m}$ wavelengths and thus are competitive to InAs/GaAs system which, however, can also be made to emit at telecom wavelengths by

*corresponding author; e-mail: Grzegorz.Sek@pwr.edu.pl e.g. using additional strain-reducing layer to operate up to even $1.5 \mu \mathrm{m}[7,8]$. While $\mathrm{InP}$ based nanostructures have been widely studied around $1.55 \mu \mathrm{m}$ [9-11], there is still not much information about their possible operation close to $1.3 \mu \mathrm{m}$, required for the transmission in the O-band telecommunication range.

The InAs/InGaAlAs/InP quantum dash (QDash) system is characterized by elongated in-plane geometry of the nanostructures along [1-10] direction with approximately triangular cross-sectional shape and high areal density $\left(>10^{10} \mathrm{~cm}^{-2}\right)$ [12]. The sample structure has been grown by EIKO gas source molecular beam epitaxy system using S-doped InP (001) substrates. The nominal thickness of the InAs layer was $0.8 \mathrm{~nm}$ which corresponds to the emission range from 1.20 to $1.33 \mu \mathrm{m}$ due to geometrical and compositional fluctuations. The sample has been processed and there have been etched mesas down to $400 \times 200 \mathrm{~nm}^{2}$ size in order to limit the number of the optically probed nanostructures.

In this work we investigate single QDashes, focusing on the properties of excitonic complexes such as binding energies, fine structure splitting, polarization anisotropy of emission, dynamics of the radiative processes and the photon statistics of emission. The experiments have been carried out within a high spatial $(1 \mu \mathrm{m})$ and spectral $(20 \mu \mathrm{eV})$ resolution micro-photoluminescence $(\mu P L)$ se- 
tup equipped with InGaAs detector array, superconducting NbN single photon counting detectors or infrared streak camera system for time-resolved photoluminescence (TRPL) measurements, and photon autocorrelation measurements in a Hanbury-Brown and Twiss (HBT) configuration. All experiments have been performed at temperature of $5 \mathrm{~K}$. The sample has been excited non-resonantly with a continuous wave laser line at $660 \mathrm{~nm}(\mu P L$ and HBT) and with a train of $120 \mathrm{fs}$ long pulses at $830 \mathrm{~nm}$ (TRPL) of $76 \mathrm{MHz}$ repetition rate, focused on the sample by long working distance and high numerical aperture microscope objective $(N A=0.4)$. We also used a cryostat with superconducting magnet that generates magnetic field up to $5 \mathrm{~T}$ and polarization resolved analysis has been performed by using a half-wave plate situated in front of a linear film polarizer.

\section{Results and discussion}

Figure 1 shows examples of $\mu P L$ spectra taken for $1 \mu \mathrm{W}$ excitation power and magnetic field of $2 \mathrm{~T}$ oriented in the Voigt configuration along [110] crystallographic direction of the sample. The polarization resolved spectra present various splittings which allow to preliminarily distinguish between the lines of charged and neutral complexes, while mixing effect of bright and dark configurations induced by magnetic field results in quadruplet or triplet of transitions for charged exciton [13, 14], and doublets for exciton and biexciton states. Thus, the observed triplet on the lower energy side reveals a trion character of this emission. As the dark exciton states $X_{D}$ are typically lower in energy than the bright ones in such nanostructures [15], the higher energy transition corresponds to exciton and the lower energy one to biexciton. Based on that, the bright-dark states splitting is equal to $440 \mu \mathrm{eV}$, the binding energies of biexciton and trion are $-3.1 \mathrm{meV}$ and $-5.6 \mathrm{meV}$, respectively. Strongly bound state of the charged exciton suggests this is the negatively charged trion [16-18], which could be expected for nanostructures deposited on InP substrate being unintentionally $n$-doped with substantial concentration of the excess electrons. Typically, as for small quantum dots, one would expect that the $X X_{D}$ PL line has significantly lower intensity than $X_{D}$ one due to the spin relaxation from bright to dark exciton which populates the $X_{D}$ states. Considering the almost equal intensities of $X_{D}$ and $X X_{D}$ transitions in our case, we could have some indications on spin-flip coupling of bright and dark exciton states, which induces a transition from dark to bright and is mediated by spin-orbit interactions and acoustic phonons due to the higher lateral extension of the QDash than for e.g. InAs QDs [19-21]. This is however unclear yet and needs further investigation.

In Fig. 2a, we present results of TRPL experiment performed on the same excitonic complexes. The photoluminescence decays have been measured at average power of $50 \mu \mathrm{W}$ of the pulsed excitation, showing exponential decay times $\left(\tau_{P L}\right)$ of $2.2,1.4$, and $2.1 \mathrm{~ns}$ for $X, X X$, and

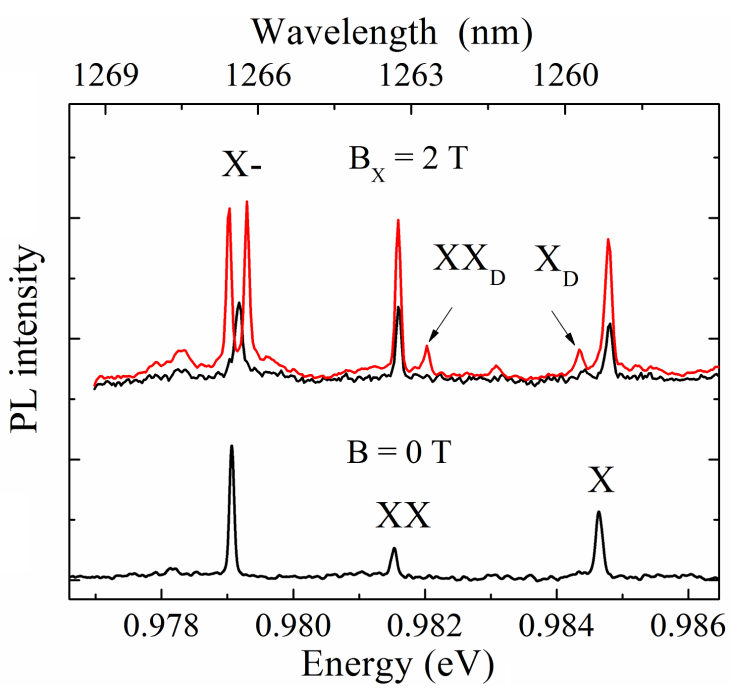

Fig. 1. Microphotoluminescence spectra taken from a non-resonantly excited single quantum dash showing exciton, biexciton and negative trion, which have been identified by the characteristic trion splitting and dark biexciton and exciton states in the in-plane magnetic field of $2 \mathrm{~T}$.

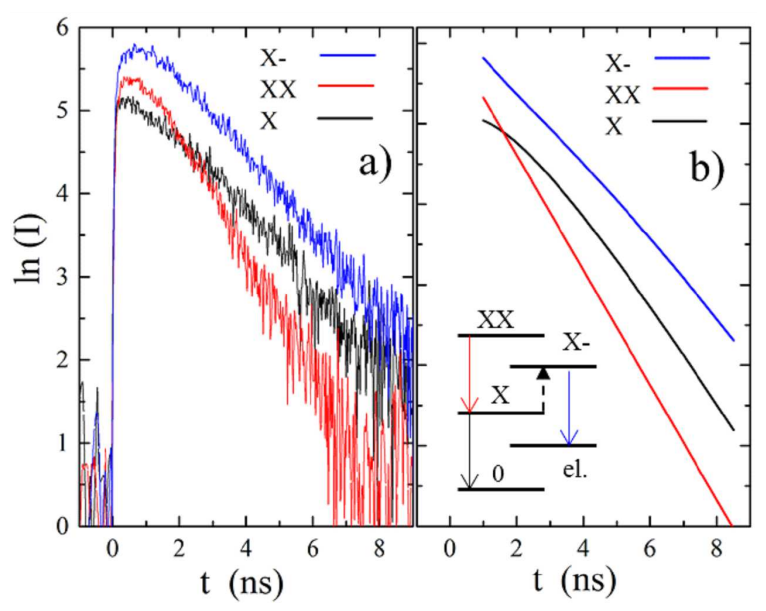

Fig. 2. (a) Photoluminescence decay of excitonic complexes measured from a single QDash and (b) the solution of the rate equation model for the radiative relaxation in which the electron capture process and thus the transition from $X$ to $X$ - state is allowed.

$X$ - lines, respectively, giving the ratios of $\frac{\tau_{X, P L}}{\tau_{X X, P L}} \approx 1.6$ and $\frac{\tau_{X, P L}}{\tau_{X-, P L}} \approx 1$. It is important to note that the obtained ratios are different than very often used values in simplified considerations when neglecting the excitonic states configuration mixing (i.e. $\frac{\tau_{X}}{\tau_{X X}}=2$ and $\frac{\tau_{X}}{\tau_{X-}}=1$ ), which is expected for the dashes with decreased confined levels' splitting. The PL decays can be perturbed by the presence of the higher order excitonic complexes, as the proximity of the $p$-shell is estimated to be lower than $20 \mathrm{meV}$ in these structures [22]. Moreover, the dark excitonic states and spin-flip processes may occur [23]. 
In addition, we cannot neglect the influence of the excess carriers on entire kinetics which has evidently been the case for the excitation power dependence in the cwexcitation as shown elsewhere [18].

Our understanding of the dynamics in this system has been supported by the obtained agreement between the simulated relaxation and the experimental results which is shown in Fig. 2b. In this case, we assumed the Poissonian statistics of the initial occupation of the five-level system as $n_{k}(0)=\frac{g^{k} e^{g}}{k !}$, where $g$ is related to the number of electron-hole pairs captured after the laser excitation pulse and $k$ is the state order equal to 0 for ground state and single electron state, 1 for $X(X-)$ and 2 for $X X$. Next, we introduced the increased probability of the system being negatively charged by setting the imbalance of the initial occupation $n_{e}(0)=N_{e} n_{0}(0)$ and $n_{X-}(0)=N_{e} n_{X}(0)$, where the indices $e(X-)$ and $0(X)$ describe the electron (trion) and ground (exciton) states, respectively. The $N_{e}>1$ reflects the influence of the excess electrons in the system and thus we found the best agreement by using $N_{e}=3$. It results in a relative difference between the intensities of $X$ and $X$ - in the quasi-equilibrium state after electron-hole pair capture process, i.e. the maximum intensity after the laser pulse excitation. Besides the imbalanced initial occupation, we additionally allowed a transition from $X$ to $X-$ due to the possible electron capture during relaxation process from $X X$, which may be the case due to residual electron concentration which can influence the observed dynamics. This electron capture rate has been optimized to $g_{e}=0.3 \mathrm{~ns}^{-1}$ in order to fit the experimental decays properly. In this model, we used similar lifetimes' values as observed in the TRPL experiment for $X, X X$, however $X$ - lifetime has been fitted to $\tau_{C X}=1.5 \mathrm{~ns}$ which is different than the decay time observed in the $X$ - photoluminescence of $\tau_{C X, P L}=2.1$ ns. Such extension of $\tau_{C X, P L}$ is well reproduced in the assumed relaxation scheme by added population channel from $X$ to $X-$ state and supports the considered interpretation.

Radiative decay of all the excitonic complexes has further been analyzed in terms of the parameter settings, as shown in Fig. 3. In the first case we considered a variation of the charged exciton lifetime $\tau_{C X}$ from $1.3 \mathrm{~ns}$ to $1.7 \mathrm{~ns}$ which induces a slope change of the $X$ - decay. Next, we tailored the imbalance of the initial occupation by setting $N_{e}=1,3,5$. We have found that the initial occupation may affect both the maximum intensity after the pulse and the slope of the $X$ - decay. These parameters have an impact on the $X$ and $X X$ decays. Last parameter studied here is the electron capture rate $g_{e}$ which is tuned from $0.1 \mathrm{~ns}^{-1}$ to $0.9 \mathrm{~ns}^{-1}$ and it causes significant change in both the $X$ and $X$ - decays leaving $X X$ unchanged. For the lowest $g_{e}$ value the exciton emission is extended in time while the charged exciton decays faster. In case of high $g_{e}$ we observe faster $X$ decay and longer $X$ - decay with some nonlinearity seen (in the log-log scale) during first $2 \mathrm{~ns}$. These simulations demonstrate that in order to reproduce the experimentally observed decays of exci-

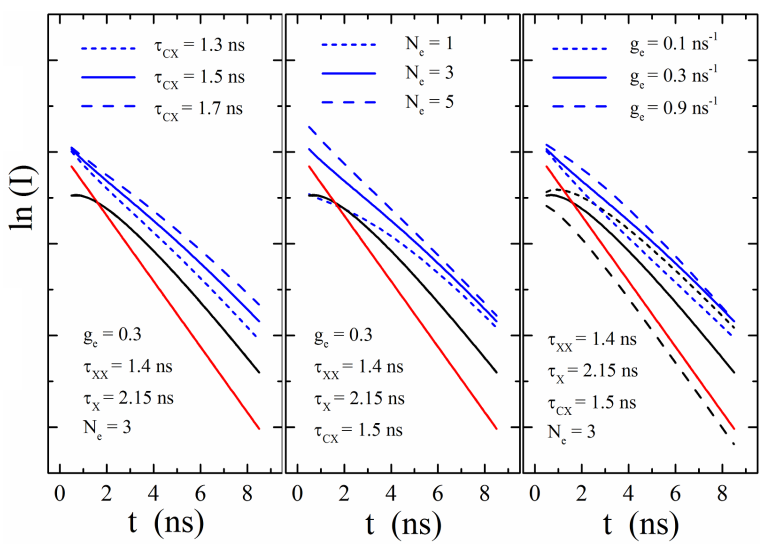

Fig. 3. A series of simulated relaxations for excitonic complexes showing the influence of variations applied to the $X$ - lifetime, initial occupation and electron capture rate.

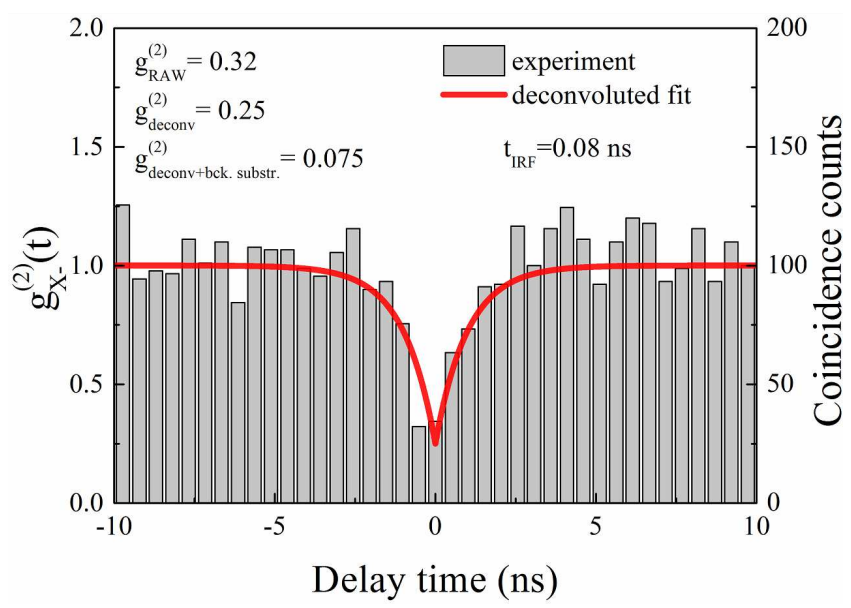

Fig. 4. Autocorrelation measurements of the negatively charged exciton showing clear antibunching, namely $g^{2}(0)$ value below 0.5 is observed in a raw data points. After deconvolution with the IRF we determined best matching for $g^{2}(0)=0.25$. Additional background subtraction reduces $g^{2}(0)$ to $\approx 0.075$.

tonic complexes from single QDash, we can consider the 5 -level rate equation model parametrized with decay lifetimes, initial occupation and the additional single carrier capture rate included in the radiative relaxation kinetics which can significantly influence the decays of excitonic complexes.

Eventually, the photon autocorrelation measurements have been performed for the negatively charged exciton emission line. The experimental results presented in Fig. 4 show a clear antibunching which is the fingerprint of the sub-Poissonian distribution of the emission events and thus qualifies such single QDashes as single photon sources operating within the range of the O-band telecommunication spectral range. The criterion of a single photon source of $g^{2}(0)<0.5$ has been obtained 
for the as-measured data already, namely $g^{2}(0)=0.32$. The fitting including the instrument response function of $t_{I R F}=0.08 \mathrm{~ns}$ gives $g^{2}(0)=0.25$, and after background subtraction $g^{2}(0)$ value drops down to approximately 0.075 .

\section{Conclusions}

Experimental study of single quantum dash photoluminescence allowed for identification of various excitonic complexes in the spectral range close to $1.3 \mu \mathrm{m}$. We focused on the complex kinetics of the radiative recombination observed in the time-resolved measurements. We compared the photoluminescence decays with a fivelevel rate equation simulation showing a significant impact of both the electron capture process and the favored occupation of the charged states on the decay times. More careful analysis exhibits a significant change in the decays of the negatively charged and neutral excitons, and thus extended trion state lifetime than the decay time observed in experiment. This could be related to the residual, unintentional high concentration of the excess electrons possible in quantum dash system. Finally, we demonstrated photon autocorrelation data for the negatively charged exciton which revealed a clear sub-Poissonian statistics being an evidence single photon emission from such nanostructures. All these results give an insight into understanding of the optical properties of InAs/InGaAlAs/InP quantum dashes and demonstrate their potential as a competitive solution to commonly considered GaAs-based quantum dots to be applied as sources of single or entangled photons in the short range quantum communication systems.

\section{Acknowledgments}

This research was supported by the National Science Centre within Grant No. 2011/02/A/ST3/00152.

\section{References}

[1] P. Michler, A. Kiraz, C. Becher, W.V. Schoenfeld, P.M. Petroff, L. Zhang, E. Hu, A. Imamoglu, Science 290, 2282 (2000).

[2] A.J. Shields, E. Limited, C.S. Park, Nat. Photon. 1, 215 (2007).

[3] S. Strauf, N.G. Stoltz, M.T. Rakher, L.A. Coldren, P.M. Petroff, D. Bouwmeester, Nat. Photon. 1, 704 (2007).

[4] N. Akopian, N. Lindner, E. Poem, Y. Berlatzky, J.E. Avron, D. Gershoni, B.D. Gerardot, P. Petroff, Phys. Rev. Lett. 96, 130501 (2006).

[5] R.M. Stevenson, R.J. Young, P. Atkinson, K. Cooper, D.A. Ritchie, A.J. Shields, Nature 439, 179 (2006).
[6] E. Waks, K. Inoue, C. Santori, D. Fattal, J. Vučković, G.S. Solomon, Y. Yamamoto, Nature 420, 762 (2002).

[7] E. Goldmann, S. Barthel, M. Florian, K. Schuh, F. Jahnke, Appl. Phys. Lett. 103, 242102 (2013).

[8] J. Kettler, M. Paul, F. Olbrich, K. Zeuner, M. Jetter, P. Michler, M. Florian, C. Carmesin, F. Jahnke, Phys. Rev. B 94, 45303 (2016).

[9] Ł. Dusanowski, M. Syperek, J. Misiewicz, A. Somers, S. Höfling, M. Kamp, J.P. Reithmaier, G. Sęk, Appl. Phys. Lett. 108, 163108 (2016).

[10] Ł. Dusanowski, M. Syperek, P. Mrowiński, W. RudnoRudziński, J. Misiewicz, A. Somers, S. Höfling, M. Kamp, J.P. Reithmaier, G. Sęk, Appl. Phys. Lett. 105, 21909 (2014).

[11] G. Saint-Girons, N. Chauvin, A. Michon, G. Patriarche, G. Beaudoin, G. Bremond, C. Bru-Chevallier, I. Sagnes, Appl. Phys. Lett. 88, 133101 (2006).

[12] A. Sauerwald, T. Kümmell, G. Bacher, A. Somers, R. Schwertberger, J.P. Reithmaier, A. Forchel, Appl. Phys. Lett. 86, 253112 (2005).

[13] J.J. Finley, D. Mowbray, M.S. Skolnick, A. Ashmore, C. Baker, A. Monte, M. Hopkinson, Phys. Rev. B 66 , 153316 (2002).

[14] D.N. Krizhanovskii, A. Ebbens, A.I. Tartakovskii, F. Pulizzi, T. Wright, M.S. Skolnick, M. Hopkinson, Phys. Rev. B Condens. Matter Mater. Phys. 72, 161312 (2005).

[15] P. Mrowiński, A. Musiał, A. Maryński, M. Syperek, J. Misiewicz, A. Somers, J.P. Reithmaier, S. Höfling, G. Sęk, Appl. Phys. Lett. 106, 53114 (2015).

[16] M.F. Tsai, H. Lin, C.H. Lin, S. Di Lin, S.Y. Wang, M.C. Lo, S.J. Cheng, M.C. Lee, W.H. Chang, Phys. Rev. Lett. 101, 267402 (2008).

[17] S. Rodt, A. Schliwa, K. Pötschke, F. Guffarth, D. Bimberg, Phys. Rev. B 71, 155325 (2005).

[18] P. Mrowiński, M. Zieliński, M. Świderski, J. Misiewicz, A. Somers, J.P. Reithmaier, S. Höfling, G. Sęk, Phys. Rev. B Condens. Matter Mater. Phys. 94, 115434 (2016).

[19] K. Roszak, V.M. Axt, T. Kuhn, P. Machnikowski, Phys. Rev. B Condens. Matter Mater. Phys. $\mathbf{7 6}$, 195324 (2007).

[20] J. Johansen, B. Julsgaard, S. Stobbe, J.M. Hvam, P. Lodahl, Phys. Rev. B 81, 81304 (2010).

[21] M. Gawełczyk, P. Machnikowski, Semicond. Sci. Technol. 32, 45005 (2017).

[22] A. Musiał, P. Kaczmarkiewicz, G. Sęk, P. Podemski, P. Machnikowski, J. Misiewicz, S. Hein, S. Höfling, A. Forchel, Phys. Rev. B Condens. Matter Mater. Phys. 85, 35314 (2012).

[23] M. Munsch, J. Claudon, J. Bleuse, N.S. Malik, E. Dupuy, J.-M. Gérard, Y. Chen, N. Gregersen, J. Mørk, Phys. Rev. Lett. 108, 077405 (2012). 